\title{
La Unión de Universidades de América Latina y el Caribe, y su idea de universidad latinoamericana
}

\author{
Marcial Antonio Rubio Correa \\ Ex rector de la Pontificia Universidad Católica del Perú
}

\begin{abstract}
Hemos tomado estas ideas de la Declaración Final del Encuentro denominado Las Universidades latinoamericanas ante los rankings internacionales: impactos, alcances $y$ límites, fechada el 18 de mayo de 2012. El texto nos fue proporcionado por UDUAL.
\end{abstract}

La Unión de Universidades de América Latina y el Caribe (UDUAL) tiene un concepto de universidad latinoamericana particular, enraizado en nuestro continente y nuestra diversidad cultural. Ve a la universidad no como una torre de marfil sino como una entidad de excelencia académica, pero entretejida con la realidad social que la alberga y con sus necesidades. No cree que sólo se trate de la excelencia académica sino también de un compromiso intenso con su medio y, todo ello, en el contexto de una gran aceleración de la globalización y la interconexión.

Es como si la universidad latinoamericana debiera ser un puente, un canal de conexión entre los avances del mundo y las posibilidades de nuestras sociedades, entre el desarrollo del conocimiento universal y nuestra capacidad de aprender y crear.

\section{La educación como bien público social}

La UDUAL reconoce a la educación como un bien público social según se sostuvo en la Conferencia Regional sobre la Educación Superior, llevada a cabo en Cartagena de Indias, Colombia, el año 2008. Lo que atañe a la educación en general incluye, entre sus especies, a la educación superior universitaria.

En el mundo contemporáneo se ha dicho, también, que la educación es un bien común correspondiente al interés general de la sociedad. Más allá de las diferencias que podamos encontrar entre los dos conceptos citados, nos interesan en este punto las similitudes que, en el terreno educativo, son significativas. Ambas expresiones tienen como punto de partida que la educación es un derecho de las personas y de los pueblos a los que ellas pertenecen porque sus beneficios llegan tanto al plano individual en cada ser humano, como al colectivo de la sociedad: la persona educada crece espiritualmente y también en sus capacidades laborales, lo que colabora con ella y su familia en lograr una mayor realización y un mejor nivel de vida. Al mismo tiempo, una sociedad de personas con educación extensiva es 
un grupo humano con más capacidades colectivas y mejores posibilidades de conjunto. Estos son conceptos de extensa difusión que no pretendemos ahora fundamentar. Sin embargo, son alternativos a la idea de la educación como mercancía y a la consideración del alumno como consumidor de ella. Consiguientemente en el plano de la educación superior, la educación como bien público social excluye la obtención de utilidades por un dueño de la institución educativa, convertida en sociedad de capitales.

La UDUAL, como red institucional, está conformada exclusivamente por universidades y otras instituciones de educación superior sin fines de lucro. Es una opción institucional y, desde nuestro punto de vista, le da identificación y consistencia.

La educación para UDUAL es un servicio al ser humano que estudia para crecer integralmente como persona y como ciudadano. La institución que le brinda educación tiene una finalidad social, colectiva y formativa, en sus diversas dimensiones: no lucra ni da utilidades a nadie en ese proceso. El dinero que recibe es para financiar más y mejor educación en un círculo virtuoso de mejora continua. Estas características dan a la UDUAL un sello propio y, a sus instituciones miembros, una identidad compartida que les permite actuar conjuntamente con mutua confianza y beneficio. Existe una homogeneidad de pensamiento y de praxis sobre la tarea educativa que nos es común

\section{Universidad institucionalmente autónoma}

La autonomía universitaria es una bandera permanente de la UDUAL, una connotación de identidad y un camino de realizaciones permanentes. Sólo una universidad autónoma puede prestar genuinamente el servicio público social educativo porque, si le imponen su quehacer, las órdenes tendrán sesgos incompatibles con el diálogo, la discrepancia y el desarrollo dialéctico del conocimiento humano.

Esta característica constitutiva de las universidades nació por contraposición al poder del Estado. Como un esfuerzo de autodirigirse académicamente para diseñar de la mejor manera su tarea: son los académicos quienes tienen más acceso al conocimiento y, por tanto, quienes mejor pueden organizarlo para transmitirlo (enseñanza) y para hacerlo crecer (investigación).

Sin embargo, modernamente, la autonomía universitaria no es sólo contra el poder del Estado que pretenda mandar lo que se deba enseñar. Tiene dimensiones académicas, económicas, administrativas, de regulación y de gobierno. Cada una de ellas se ha vuelto más difícil de elaborar cabalmente, contagiada de la velocidad y las complejidades de la vida moderna. Por ello la autonomía ha ido cambiando progresivamente, pero no en el sentido de abandonar determinadas significaciones para adoptar otras nuevas, sino en el de enriquecerse, de acumular nuevas formas de ser autónomo sin perder las primeras.

Esto requiere un repensar permanente y creativo, que ajuste la necesaria autonomía universitaria al contexto social cambiante. La UDUAL tiene para ello un observatorio y una actividad de discusión y publicación permanente. Es una de las instituciones latinoamericanas que, de manera más 
constante y pugnaz, trabaja en el desarrollo y promoción de la autonomía universitaria en nuestro espacio común ibero-latinoamericano. En este sentido ha dicho:

La autonomía universitaria ha sido un tópico identitario de la Unión de Universidades de América Latina y el Caribe (UDUAL), desde que en 1949 se constituyera, a convocatoria del Dr. Carlos Martínez Durán, como un organismo de las instituciones de educación superior en la región. El principio rector de la lucha por ampliar los derechos universitarios, preservar su autonomía de pensamiento, académica y administrativa, ha enfrentado temporales adversos, movimientos de impulso y acechanzas permanentes. Es una historia de contratiempos y realizaciones, pero sobre todo de construcción de la identidad universitaria en América Latina".

\section{Un espacio iberoamericano común de conocimiento}

La UDUAL y sus instituciones miembros pertenecemos a un espacio internacional definido desde hace casi quinientos años, cuando la Universidad de Salamanca fue modelo de creación de las universidades que, luego, pasamos a llamarnos latinoamericanas. Hoy Salamanca es una de varias universidades ibéricas asociadas a la UDUAL, para dar consistencia a este espacio común en dos orillas del Atlántico.

Somos universidades latinoamericanas e iberoamericanas, pero no aisladas por las fronteras. En el mundo universitario de siempre, pero con gran intensidad en la actualidad. Las universidades compartimos recursos y espacios para que profesores y estudiantes vayan y vengan entre nosotras a fin de tener experiencias nuevas de vida intelectual, de compartir y acrecentar conocimientos, así como de crear lazos de mutua colaboración en la gigantesca tarea de aumentar el acervo del conocimiento humano. Por razones históricas y culturales los latinoamericanos, entre nosotros, y con los ibéricos, compartimos dos lenguas romances que nos acercan en el trabajo intelectual y nos facilitan la comunicación. Por ello, muchos estudiantes latinoamericanos viajan a la península ibérica a proseguir sus estudios de posgrado.

La UDUAL busca institucionalizar este espacio común y compartirlo con creces, hacerlo algo equivalente a una gran universidad común iberoamericana. Es un proyecto consistente con la internacionalización de la vida académica en el mundo, pero con caracteres particulares de comunidad que lo hacen especialmente familiar y extenso.

Concebir un espacio común cualquiera que éste sea, y desarrollarlo, son dos tareas diferentes porque lo primero requiere creatividad y lo segundo un esfuerzo continuado e intenso. La UDUAL ha puesto ambas cosas para lograrlo.

\section{Una universidad vinculada a su sociedad}

La Unión de Universidades de América Latina y el Caribe propugna una universidad latinoamericana vinculada estrechamente, y de diversas maneras, a la sociedad dentro de la cual existe. 
Los pueblos latinoamericanos tenemos un pasado precolombino propio, con una cultura rica y desarrollada apropiadamente para sus condiciones de tiempo y espacio. El arribo de Europa a América fue, para esas culturas y sus gentes, un proceso traumático de conquista e instauración de un régimen colonial cuyas líneas generales se conocen. La independencia trajo la autonomía política de nuestros Estados nacientes, pero la sociedad mantuvo las fracturas que se desarrollaron en la época colonial.

\section{La historia y la raíz de nuestros pueblos}

Somos mestizos, herederos de nuestro pasado tanto el anterior a las conquistas como el colonial, y nos construimos como naciones en un proceso progresivo de avances y conflictos. Los pueblos latinoamericanos tenemos conciencia de que esta construcción está a medio hacer, que se ha producido con errores significativos que hay que corregir y que debe proyectarse a un futuro de diversidad y mutuo respeto.

Cada una de nuestras universidades tiene una tarea que hacer en este proceso. Desde las ciencias exactas hasta las humanidades, poseen conocimientos indispensables para tejer la urdimbre mutuamente respetuosa de nosotros mismos. Es un esfuerzo por esclarecer, explicar, llevar hilos conductores y proponer los cambios y proyectos sociales globales que nos conduzcan a la integración y potenciación de nuestros pueblos. La literatura académica se ocupa intensamente de esta tarea. Los académicos son columnistas de opinión y comentaristas de los medios de comunicación. Con su diversidad, sus teorías y apreciaciones, colaboran a construir esta realidad y a darle rumbo. La potencia del conocimiento acumulado en nuestras universidades es significativa para contribuir al proyecto común de nuestros pueblos.

Somos sociedades multiculturales y todavía tenemos segregación y discriminación entre nosotros. Existen declaraciones jurídicas y políticas de igualdad pero, en la vida cotidiana, en la ideología común, todavía existen multitud de diferencias que conducen a considerar de mayor o menor situación a las personas. Son códigos de segregación que se transmiten por vías informales y que se asumen porque, en realidad, el trato de iguales es una forma superior de concepción espiritual al que hay que acceder. Las universidades, con su saber, pueden colaborar decisivamente a desmontar los mecanismos ideológicos de la segregación y, con ello, a desarrollar una multiculturalidad con mutuo respeto. Existen grandes esfuerzos ya en camino entre nosotros los universitarios, pero se necesita un redoblado esfuerzo para cambiar la situación. Por sus conocimientos y capacidades, las universidades tienen una posición privilegiada para ser levadura en la masa, si deciden asumir ese reto.

Un aspecto particular de la multiculturalidad segregada es la cantidad de lenguas que existen en Latinoamérica. Durante largo tiempo se trató de suprimir las lenguas aborígenes para universalizar el castellano o el portugués, respectivamente, en sus territorios. La resistencia cultural, sin embargo, lo hizo imposible y es una demostración de los errores de la segregación cultural y, dentro de ella, específicamente de la idiomática. El progreso de nuestras distintas lenguas hacia su comprensión extensiva y 
hacia la capacidad de expresarse por escrito para ser leídas, es típicamente universitario: un trabajo de alta especialización en investigación. Se hace, pero se debe multiplicar. Es parte de la importancia trascendental de las humanidades en el mundo contemporáneo. A ellas nos referiremos luego.

\section{Estudio de los problemas atávicos}

En adición al problema de la segregación cultural ya mencionado antes, están también la pobreza, la desigualdad, la inseguridad y la corrupción, entre otros. No son problemas privativos de América Latina, pero tienen mucha importancia dentro de ella.

La pobreza y la desigualdad, distintas pero confluentes, son asuntos sociales estructurales para los cuales hay recetas de tratamiento, pero son muy difíciles de abordar por el juego de intereses, la cultura individualista predominante y las estructuras generales de organización de la sociedad.

En su solución colaboran todas las disciplinas del saber y, por ello, son un campo privilegiado de la investigación y la enseñanza universitaria: casi no existen otras instituciones distintas a las universidades, con la riqueza y variedad del conocimiento que ellas poseen. Desde luego es necesario un trabajo teórico para abordar cada uno de estos temas, pero también uno aplicado: deducir de lo teórico, las recetas de solución para aplicarlas a la realidad. Los dos ámbitos pertenecen por igual al quehacer de las universidades y, la eliminación de la pobreza y la desigualdad, son tareas de la más alta importancia para las sociedades latinoamericanas.

La inseguridad es un problema multiforme. Está el entorno delictivo que tiene como caldo de cultivo, precisamente, la convergencia de la pobreza y la desigualdad. Tiene también un componente de ausencia de formación cívica y, no menos importante, una incapacidad estructural del Estado para combatirla y, si es necesario, para apoyarse en la sociedad con tal propósito. La inseguridad delictiva tiene una amplia problemática social, dentro de la cual los estudios sociales universitarios juegan un importante papel: hay que desentrañar las causas con detalle y diseñar las soluciones de diverso tipo para combatirla. La represión pura ha demostrado siempre su incapacidad de solucionar el problema. La solución se encontrará necesariamente en una diversidad de medidas sociales, económicas y políticas, que permitan abordarla con éxito. Hay muchas sociedades, ricas y pobres, que lo lograron. La delincuencia no es invencible. Hay que pensar cómo superarla, y pensar es consustancial a las universidades, aunque no sólo a ellas.

Está también la inseguridad propia de los fenómenos naturales para los cuales la ciencia y la tecnología han desarrollados soluciones que, sin embargo, no son aplicadas. Quien esto escribe, peruano, ve con frecuente periodicidad a los mismos ríos asolar las mismas ciudades año tras año con las crecidas que ocurren con las lluvias veraniegas, o con la presencia de El Niño. Es como si no hubiera capacidad de asegurar los cauces, o como si no se supiera medir cuál es el refuerzo a prueba de la confluencia de las más duras condiciones. Como si un año no bastara para hacer los trabajos mínimamente necesarios.

Lo que sirve para las crecientes de los ríos se aplica a los huracanes, a los movimientos sísmicos, al frío invernal y al calor veraniego, entre otras cir- 
cunstancias amenazantes. El ser humano no puede impedir los fenómenos naturales, pero puede mitigar sus efectos con ciencia, tecnología, organización y políticas adecuadas. Las universidades tenemos mucho por delante en cada uno de estos temas. La UDUAL apoya activamente este ámbito de trabajo con su Red Universitaria de Latinoamérica y el Caribe para la Reducción del Riesgo de Desastres (REDULAC/RRD).

De la corrupción se conoce poco. Es un tema difícil de investigar. Un reto a la creatividad metodológica universitaria. La tecnología moderna permite averiguaciones que antes eran imposibles de hacer sobre ella pero, al mismo tiempo, pone en manos de los corruptos instrumentos para realizar mejor sus propósitos y borrar huellas e indicios. Es una metamorfosis de la vieja alegoría del árbol del bien y del mal de la ciencia. Las universidades iberoamericanas podemos utilizar los medios que nos pone la UDUAL en común, para establecer un marco colaborativo de lucha contra la corrupción, utilizando nuestro propio instrumental de enseñanza e investigación.

Existen vías preventivas y sancionadoras para combatir la corrupción. Ambas están poco elaboradas. La creatividad institucional y normativa de las universidades puede colaborar a desarrollarlas. Es otro servicio que las universidades podemos prestar a la organización social.

\section{Los derechos humanos}

Los derechos humanos son un elemento de la vida contemporánea que requiere de un desarrollo espiritual superior: reconocer iguales, reconocer personas libres y compartir con ellas los espacios de nuestros derechos es un grado de civilización superior de la humanidad que se va alcanzando progresivamente.

Las universidades debemos explicar a nuestras sociedades cómo era la vida sin derechos humanos, hace menos de cien años. Tenemos que mostrar la superioridad de la vida contemporánea y, al mismo tiempo, hacer notar que la solución de los conflictos interpersonales e intergrupales por las vías institucionales de la aplicación jurisdiccional de los derechos fundamentales, constituyen una calidad de vida superior para cada uno de nosotros.

Los derechos tienen que ser explicados y difundidos. Todo esfuerzo es necesario en las condiciones actuales en las que marcados sectores sociales los apoyan o los consideran negativos. El consenso sobre los derechos humanos está aún por ser alcanzado aunque, es verdad, hay una creciente aceptación de ellos.

Un aspecto particular es el de los derechos sociales, calificados generalmente como derechos programáticos en la teoría, lo que da a muchos políticos la facilidad de sostener que no son exactamente exigibles. La idea es que se trata de una aplicación progresiva sin paso atrás. Nuevamente, la universidad tiene un papel central en mostrar la importancia de los derechos sociales y la necesidad de su afianzamiento en la sociedad. No es fácil si tomamos en cuenta la cultura predominantemente individualista contemporánea. Es una tarea a contracorriente, pero fundamental porque, entre otras cosas, colabora a eliminar la pobreza y las grandes desigualdades hoy existentes. 
Otro aspecto importante de los derechos humanos es la igualdad de género que requiere impulso en América Latina, poblada por una ideología machista que es preciso cambiar. La acción positiva y la existencia de políticas progresivas, en este sentido, son un terreno en el que las universidades tienen importantes labores por delante, dentro y fuera de ellas mismas.

Finalmente, entre otros muchos, está el acceso a la justicia: un derecho constitutivo del Estado contemporáneo que debe ser reforzado en la inmensa mayoría de nuestros países a través de una sustantiva reforma del sistema judicial. Es un típico problema estructural porque es necesario reformar viejos moldes de administración de justicia, pero también funcional porque la creatividad para mejorar los procedimientos, y acelerar los procesos, es fundamental para lograr una real pacificación de la sociedad a través de la judicatura. El diseño de ambos aspectos, estructural y funcional, debe tener un aporte importante del mundo universitario latinoamericano.

Desarrollar los derechos humanos y difundir su conocimiento son dos tareas pendientes en América Latina y el concurso de todas las instituciones, entre ellas la universitaria, es muy importante para lograr éxitos en la conciencia y en la aplicación de los derechos humanos.

\section{El cuidado del medio ambiente}

América Latina es un subcontinente con gran riqueza medioambiental en todos sus sentidos. Es una reserva de alcance mundial. Nuestra población tiene el deber de protección de esta riqueza, no sólo para sí, sino para toda la humanidad.

La protección del medio ambiente es un conocimiento y también una praxis. Ambas cosas deben ser aprendidas. La enseñanza del cuidado y desarrollo del medio ambiente adecuado es una indudable prioridad de nuestras universidades. También lo es desarrollar entre los miembros de nuestras comunidades universitarias las formas prácticas de vida que colaboran a ello. El campus universitario donde existe es una buena escuela para lograrlo.

La actividad de investigación en el área del medio ambiente y de la variedad de vida animal y vegetal, tiene más cosas por hacer que las ya hechas. Al propio tiempo, las dimensiones de la tarea superan largamente lo que las universidades aisladas unas de otras podemos hacer. La UDUAL estimula el trabajo colectivo en redes temáticas, y la campaña de Universidades Sostenibles y Resilientes ha desarrollado trabajos internacionales conjuntos, entre otros ámbitos, en el de "la articulación con otras agendas internacionales, regionales, nacionales y locales, especialmente con las de sostenibilidad, cambio climático, asistencia humanitaria, hábitat y otras que la institución priorice según su ubicación geográfica”.

Una labor que nuestras instituciones académicas pueden realizar, en pro de la defensa del medio ambiente, es la concientización general a través de campañas informativas y el apoyo a los diversos niveles de gobierno nacional para comprometerlos en la tarea. Esto es tanto más importante si consideramos que en el contexto internacional existen políticos de países poderosos que desdeñan los avisos que la ciencia da sobre el deterioro de 
las condiciones de vida del planeta, en pos de conseguir ventajas económicas de corto plazo.

\section{El proyecto de desarrollo nacional}

Nuestros países latinoamericanos tienen proyectos de desarrollo nacional que pueden ser apoyados de diversas maneras por nuestras universidades.

Primero está la concepción con base en la cual se elaboran los problemas y las prioridades que se seleccionan, así como la orientación general que se busca en función de las posibilidades de cada país. Todo ello exige un proceso racional de diseño en el que las comunidades universitarias pueden aportar con base en sus métodos de trabajo y sus conocimientos.

Luego está el desarrollo mismo del proyecto nacional con todas sus variables. En ello el aporte de investigación aplicada de la universidad puede ser vigorizador de la calidad de las propuestas. Un marco general de elaboración que integre variables, les de consistencia y mutua interacción es una colaboración igualmente deseable.

El diseño de los métodos y estructuras sociopolíticas que harán realidad el plan también tiene suma importancia, tanto a nivel nacional, como regional y local. Problemas antiguos como el centralismo, o el autoritarismo y burocratismo de las administraciones públicas, pueden ser superados con diseños creativos y modernizados. En este sentido, la UDUAL trabaja permanentemente en lograr la creciente participación de las universidades. Su Red de Desarrollo Local es uno de los recintos institucionales en el que esta labor conjunta puede ser realizada con ventajas.

Un campo en el que se abren posibilidades crecientes de desarrollo nacional es el de la relación entre la universidad y la empresa: ambas instituciones que durante mucho tiempo han tenido una vida paralela en la sociedad. Hoy hay esfuerzos de convergencia que tendrán que profundizarse y enriquecerse progresivamente. La UDUAL tiene la Red Universidad-Empresa (ALCUE), definida como un "espacio abierto para intercambiar experiencias y promover la cooperación entre las instituciones y personas del espacio ALCUE que impulsan las relaciones entre las universidades y las empresas para propiciar la innovación y el desarrollo económico-social de los países".

Las universidades también tenemos experiencia extensa de vinculación con la colectividad, especialmente con sectores postergados de la sociedad que deben ser incorporados para participar democráticamente en el diseño de los proyectos nacionales. En muchos casos es necesario hacer consultas previas a los pueblos originarios según disposiciones internacionales y nacionales. En otros es preciso hacer una labor de acercamiento de la administración estatal a las poblaciones para lograr mutua comprensión sobre la realización de los proyectos, en determinadas áreas geográficas sensibles.

\section{Una síntesis de la relación universidad sociedad}

La labor universitaria frente a su sociedad es extensa y variada, como puede apreciarse de la resumida reseña que hemos hecho. Pero es fundamental en la definición institucional de las universidades de esta región del mun- 
do. Somos instituciones enraizadas en las sociedades dentro de las cuales vivimos y propugnamos su desarrollo en muy diversos aspectos: económicos, sociales, culturales, geográficos, organizativos y, por supuesto, también en el conocimiento.

Miramos conjuntamente al pasado para entendernos mejor y proyectarnos positivamente hacia el futuro. Trabajamos en todos los ámbitos de punta de la vida social contemporánea y, también, en el pensamiento clásico de diversas épocas, como un aporte a muchos aspectos del presente. Tratamos de colaborar en los proyectos de futuro y en una mayor conciencia y espiritualidad de las personas, y de la sociedad en su conjunto. La UDUAL promueve todas estas actividades y, en muchas de ellas, tiene grupos y redes de trabajo para elaborar en común. En ese sentido, la UDUAL es una organización para el progreso de toda América Latina, dentro de un espíritu iberoamericano inscrito en nuestro pasado pero, también, proyectado hacia un mejor porvenir.

\section{Sobre el quehacer académico universitario}

Nuestras universidades, dentro de UDUAL, no solamente trabajan en los grandes problemas conceptuales de la universidad latinoamericana que hemos visto hasta aquí. El quehacer académico, propio y cotidiano de nuestras instituciones, también se enriquece en el ámbito común que ella facilita.

\section{La enseñanza y su desarrollo institucional}

La universidad latinoamericana tiene como una de sus principales tareas la de enseñanza pues educa profesionales, maestros y doctores para todas las labores especializadas que se requieren en su ambiente. También forma técnicos que colaboren en los trabajos especializados que sean necesarios, realiza educación continua para el perfeccionamiento a lo largo de la vida y difunde extensamente la cultura en la sociedad.

Hasta hace pocas décadas la labor de enseñanza universitaria era esencialmente empírica. El maestro universitario empezaba a enseñar con base en los conocimientos especializados de su profesión, pero sin una formación pedagógica que le diera instrumentos para apoyar exitosamente a sus alumnos.

En los años recientes la enseñanza superior ha empezado a ser una especialidad en sí misma, fruto del avance acelerado del conocimiento, y del ingreso de las tecnologías informáticas al aula y del consiguiente cambio de la relación profesor-alumno.

El profesor de hoy no puede enseñar de la misma forma en que le enseñaron sus maestros y la razón principal es que antes el maestro enseñaba y ahora el alumno aprende. En realidad el alumno siempre aprendió, pero se pensaba que el maestro lo imbuía de conocimiento. Hoy se sabe que el maestro acompaña el aprendizaje del alumno, cosa muy distinta a la transmisión mecánica de conocimientos.

Todo esto requiere técnicas de enseñanza que evolucionan constantemente al paso de la tecnología, así como de los descubrimientos de la neurociencia en relación con el aprendizaje humano. Para un profesor uni- 
versitario actual es preciso saber también de esto -no sólo de lo que se enseña- y aplicarlo en las nuevas aulas de clase.

El concepto mismo de aprendizaje ha evolucionado. Antes se pensaba que se producía por una acumulación de conocimientos. Hoy se constata que los conocimientos se enriquecen, evolucionan, se reconfiguran con cada nuevo aprendizaje y, también, caen en obsolescencia. Por tanto, si bien es necesario adquirir los conocimientos aceptados de hoy, también hay que aprender competencias para poder adquirir por sí mismo los conocimientos que sobre nuestra materia traiga el futuro. El profesor no sólo debe transmitir conocimientos sino desarrollar competencias en sus alumnos. Cada especialidad tiene un catálogo propio de competencias y hay otras de ellas que son comunes a todos los ámbitos del saber. El profesor tiene que estudiar para acompañar debidamente a quienes aprenden con él.

Consciente de estas necesidades, la UDUAL promueve activamente el intercambio de profesores y la evaluación de los avances del proceso enseñanza-aprendizaje en la educación superior. Sus instituciones miembros nos favorecemos de todo ello debido a la comunidad de información que la UDUAL pone a nuestro alcance. Es un entorno común en el que participan cada vez más instituciones y personas.

\section{Planes de estudio y convalidación de grados y títulos}

Un tema de creciente importancia en la universidad contemporánea es la necesidad de actualizar y evaluar integralmente los planes de estudio. Hoy se acepta que el conocimiento se duplica, en promedio, cada cuatro años y, en algunas especialidades, en menos tiempo.

Por consiguiente, decidir qué se enseña a un alumno universitario para ser competente en una determinada carrera, tiene dos problemas evidentes. El primero, que es necesario incorporar los nuevos conocimientos al plan de estudios. El segundo refiere a que, si se desea mantener el ciclo de estudios universitarios profesionales dentro de una duración de entre cinco o seis años, al tiempo que se incorpora al plan de estudios los nuevos conocimientos, es preciso retirar algunos otros. Decidir esto es tarea ardua y, muchas veces, materia de discrepancia. Adicionalmente, hoy en día un plan de estudios no es solamente una lista de conocimientos a aprender sino también comprende, dentro de sí, el método de aprendizaje que le corresponde y que es distinto según las materias, las competencias y las aplicaciones prácticas a las que conduzca el conocimiento aprendido.

Elaborar el plan de estudios es un trabajo concienzudo y en constante transformación. Para hacerlo cabalmente no sólo se necesita buscar el mejor diseño en cada universidad, sino también compartir experiencias y soluciones entre diversas universidades. Esto permite aprender de los demás con sus mejores soluciones, evitando asimismo los errores en que incurrieron. La colaboración es esencial entre las universidades para este propósito. La UDUAL es un ámbito en el que la colaboración en el diseño de nuevos planes de estudio se comparte entre las instituciones miembros que así lo desean.

Un proyecto muy interesante, desarrollado por la UDUAL durante los últimos tres años, fue el establecer condiciones de reconocimiento de grados 
y títulos entre un grupo de universidades que la integran, cada una de un país latinoamericano distinto, dentro de un número determinado de especialidades. Fue un trabajo inédito en su magnitud pues requirió que cada una de esas universidades conociera el plan de estudios integral de la carrera que dictaban cada una de sus contrapartes. Así se formaron en internet espacios de presentación y discusión de los planes a reconocer, en un esfuerzo de divulgación que ya fue, en cierto sentido, un fin en sí mismo.

El trabajo fue culminado con éxito. Los convenios respectivos se firmaron con anuencia de las autoridades de cada universidad y, desde luego, todo ello enriqueció la comprensión de sus propias materias a los profesores involucrados en la tarea. Para los graduados de cada una de estas universidades, tener la posibilidad de revalidación en otros países por los acuerdos hechos a través de la UDUAL es una ventaja de largo plazo, enorme e innegable. La UDUAL piensa ampliar el número de participantes en estos acuerdos, lo que deberá contar con la anuencia de los ya comprometidos. Los efectos benéficos crecerán.

\section{El desarrollo de la investigación}

Hasta hace poco tiempo, la inmensa mayoría de universidades de América Latina éramos universidades de enseñanza. La investigación se hacía - predominantemente- como un esfuerzo paralelo del cuerpo docente. No estaba integrada institucionalmente a la labor cotidiana.

En la actualidad varias de nuestras universidades han decidido ser de enseñanza e investigación y, algunas, van camino a ser universidades de investigación. Estos cambios requieren no solamente ponerse a investigar. Tiene que haber una reestructuración del cuerpo docente, que es el que enseñará e investigará o hará exclusivamente una de dichas tareas. Pero también será indispensable que se organice una unidad de investigación (probablemente un vicerrectorado) que disponga los detalles de cómo se hace la investigación en su universidad, cómo se estimula, cómo se financia y cómo se supervisa. En ello, compartir experiencias entre las universidades latinoamericanas, y también con universidades del mundo desarrollado, es un requisito indispensable, nuevamente, para aprovechar sus buenas ideas y evitar sus errores pasados.

El establecimiento del Espacio Iberoamericano Común de Conocimiento es una ayuda fundamental para aprovechar las experiencias latinoamericanas, y la de las universidades ibéricas, conectadas a importantes universidades del norte del mundo, muchas de ellas universidades de investigación.

Desde luego, en la UDUAL no sólo discutimos estos aspectos de la investigación. También se trata el tema de qué investigar. En la vida universitaria tiene espacio toda investigación teórica o aplicada. Las universidades no pueden sacrificar ni la una ni la otra.

Sin embargo, puede haber ciertas líneas de investigación que, de acuerdo al ser propio de cada universidad y a las condiciones del entorno en el que vive, deban ser especialmente estimuladas. Son decisiones difíciles de tomar. Nuevamente compartir ideas en el seno de la UDUAL, es una ventaja para diseñar y transitar rumbos correctos. La investigación 
teórica hace avanzar el conocimiento y por ello mismo es indispensable. Sin ella no hay mayor posibilidad de realizar una investigación aplicada de calidad suficiente.

Sin embargo, en el ámbito del compromiso con la sociedad, a la universidad le es necesario hacer investigación aplicada -de diverso tipo-para encontrar tecnologías de todas las naturalezas, que produzcan resultados concretos para el progreso de la sociedad. Algunas de nuestras universidades son prioritariamente tecnológicas. Con ellas, la UDUAL ha constituido la Red de Universidades Tecnológicas y Politécnicas de América Latina y el Caribe, una institución que permite compartir experiencias en amplia extensión dentro de este ámbito de trabajo.

Finalmente hay que tomar en cuenta el trabajo especializado en consultoría para los proyectos importantes de cada país, o de los que hagan en conjunto dos o más de ellos. A menudo requieren la conjunción de conocimientos que conviven en las universidades. Será necesario que sus miembros apliquen, al realizarlos, el conocimiento acumulado y las competencias profesionales de su propio giro con un trabajo en equipo que potencie su aporte.

\section{Sobre las humanidades y las artes}

En una época en la que algunos niegan o disminuyen el valor de las humanidades y del arte como elementos formativos, y aun de las ciencias básicas, incidiendo sólo en los conocimientos instrumentales para el trabajo, la UDUAL ha mantenido el equilibrio indispensable para la vida universitaria dando su justo valor a los diversos componentes del saber humano.

Las humanidades forjan el espíritu y desarrollan con ello a la persona, dan sentido a su vida y la proyectan en la realización de muchas maneras complementarias, todas esenciales. Los valores del ser humano como tal, y de su civismo, son obtenidos, entendidos y acrecentados con los estudios humanísticos. La universidad no debe dejar cerradas esas puertas a sus alumnos. Entreabrirlas es ya una contribución central a su ser y su felicidad futuras. Es muy significativo que entre las materias que la UDUAL eligió para hacer las convalidaciones de grados y títulos de las que hemos hablado antes, una fuera la filosofía.

La formación artística es distinta, pero de la misma naturaleza que la formación en humanidades: hace crecer el espíritu humano. No debería haber alumno universitario que, de alguna forma, no hubiera tenido una formación artística. Esto se practica en la educación básica de muchos países. No se debe interrumpir en la educación universitaria. La UDUAL tiene una Red de Integración Académica de las Artes esparcida por América Latina. Es un apoyo de gran importancia para el enriquecimiento de las unidades académicas de las universidades que participan en ella.

\section{La gestión de la universidad}

La necesidad de una gestión eficiente de las universidades ha aparecido en los últimos tiempos, al compás de la cada vez más compleja sociedad en que vivimos. No era una preocupación central en las autoridades univer- 
sitarias hace unos decenios: casi lo único importante era la seriedad de la vida académica.

Lo académico mantiene preponderancia en el trabajo universitario, pero ha crecido significativamente la necesidad de una gestión eficiente -entre otras razones- porque hoy aprender significa tener condiciones de estudio y elementos complementarios a la mano: servicios de cómputo, laboratorios, bibliotecas, hemerotecas, aprendizaje de idiomas y muchos otros recursos que requieren inversión constante de dinero. El borde de lo que cada universidad (cualquiera que ella sea) requiere gastar para mejorar el aprendizaje de sus alumnos es, en la práctica, infinito. La obtención de recursos y su disposición ordenada son esenciales para darles óptimo uso.

Por otro lado, la universidad no puede ser gestionada como si fuera una empresa productora de bienes, porque es una institución de formación de seres humanos, con procedimientos y tiempos que no son los de una línea de producción de cosas. Nos referimos por supuesto a las universidades sin fines de lucro. En ellas el concepto mismo de la gestión, y sus técnicas, tienen que adaptarse a la manera de trabajar. Así, en los últimos decenios, han aparecido planes de estudio que conducen a grados para aprender la gestión universitaria.

La UDUAL apoyada por tres de sus universidades miembro, ha establecido una Maestría en gestión de la calidad y evaluación en educación superior, destinada a formar cuadros universitarios con capacidad de desarrollar eficientemente esta labor. Es un proyecto que beneficia a muchas universidades latinoamericanas con su trabajo.

\section{A manera de conclusión}

La concepción de universidad latinoamericana que tiene la UDUAL, y por la cual trabaja arduamente como hemos visto, es la de una institución dedicada a la enseñanza como un bien público social, que está destinada a desarrollar a las personas, en sí mismas, y en todos los ámbitos del saber científico, humanístico y artístico. Excluye de la actividad educativa la finalidad de lucro. Cree y propugna que la universidad debe ser una institución autónoma en todos los sentidos necesarios para gobernarse y conducir sus actividades de la mejor manera posible -sin órdenes de fuera que cumplir- porque éstas menoscabarán la calidad de su trabajo.

Es una universidad integrada al mundo globalizado porque el conocimiento es universal y posa en todos los rincones del mundo. Dentro de esta tendencia general, la UDUAL colabora a construir un espacio iberoamericano común del conocimiento al reconocer los lazos históricos y culturales que vincula a América Latina y Europa, con las ventajas consiguientes. Con este espacio en constante construcción, la UDUAL apoya la interconexión de las universidades entre sí, su mutuo aprendizaje y un mejor servicio a sus miembros y su realidad contigua.

La universidad latinoamericana está enraizada en su sociedad. A ella sirve. La impulsa hacia el progreso, e interpreta y da sentido a su historia y cultura, en el más amplio significado de las palabras. El trabajo universitario es un instrumento útil para la extensión de la cultura de los pueblos y para la potenciación de las virtudes que tiene la multiculturalidad. 
La UDUAL concibe a la universidad como una entidad de alta calidad académica en todas sus labores, sean de enseñanza, investigación, gestión y de responsabilidad social. Hace esfuerzos por desarrollarlas con calidad teleológica y modernidad entre todas sus instituciones miembro.

Esta caracterización de nuestras universidades merece reflexión para afinar nuestra identidad común, así como para dar mejor dirección a nuestro trabajo cotidiano. La labor de UDUAL es de significativa ayuda en este sentido. 